\title{
APLICAÇÃO DE ELEMENTOS FINITOS NA ODONTOLOGIA: UMA REVISÃO DE LITERATURA
}

\author{
APPLICATION OF FINITE ELEMENTSIN DENTISTRY: \\ A LITERATURE REVIEW
}

\begin{abstract}
João Victor Couto Brito', Dulcinéia Carlos Garcia², Silas da Silva Crispim ${ }^{3}$ Jefferson David Melo de Matos ${ }^{4}$, Viviane Maria Gonçalves de Figueiredo ${ }^{5}$
\end{abstract}

\begin{abstract}
'Graduando de Odontologia, Centro Universitário UNILEÃO. Juazeiro do Norte, Ceará, Brasil. joaovcbodonto@hotmail.com ${ }^{2}$ Graduando de Odontologia, Centro Universitário UNILEÃO. Juazeiro do Norte, Ceará, Brasil. dulcygarcia@hotmail.com ${ }^{3}$ Graduando de Odontologia, Centro Universitário UNILEÃO. Juazeiro do Norte, Ceará, Brasil. silascrispim1993@gmail.com ${ }^{4}$ Graduando de Odontologia, Centro Universitário UNILEÃO. Juazeiro do Norte, Ceará, Brasil. matosjefferson19@gmail.com
\end{abstract} ${ }^{5}$ Doutora em Odontologia Restauradora. Professora no Centro Universitário UNILEÃO. Juazeiro do Norte, Ceará, Brasil. vivi_mfigueiredo@yahoo.com.br

RESUMO | Objetivo: objetiva-se com esta revisão de literatura esclarecer a aplicação da ferramenta de elementos finitos na Odontologia. Método: A revisão de literatura foi pautada em uma literatura específica e relevante, ou seja, artigos científicos, teses e livros sobre a temática abordada, utilizando as bases de dados das plataformas Pubmed.gov, Google.acadêmico, Scielo e outras bibliotecas virtuais. Considerações Finais: A Odontologia encontra no método dos elementos finitos uma forma de aprimorar a prática clínica, através de uma ferramenta computacional, para análises tensionais e estruturais nos materiais dentários e estruturas orofaciais.
ABSTRACT | Objective: Objective is with this literature review to clarify the application of finite elements in dentistry. Method: A literature review was based on a specific and relevant literature, ie, scientific articles, theses and books on the theme. This literature has been conductedon the following platforms: PubMed, Google Acadêmico, SciELO and virtual libraries. Conclusions: Finite elements is a method to improve clinical practice, through a computational tool to tension and structural analyses in dental materials and orofacial structures.

Keywords: Dentistry; Mechanic; Bioengineering.

Palavras-chaves: Odontologia; Mecânica;

Bioengenharia. 


\section{INTRODUÇÃO}

O método dos elementos finitos (MEF) originalmente foi criado para resolver problemas estruturais no âmbito da engenharia no qual avaliava o grau de deformação e tensão que um sólido sofria quando determinadas cargas eram impostas sobre ele. Seu uso tornou-se corrente durante a década de 60, quando $\circ$ mesmo sofreu uma grande evolução e o recurso foi transferido para o computador para análises geométricas arbitrárias de materiais que são sujeitos a sofrer qualquer tipo de carga'. Em geral o MEF é definido como um método matemático que utiliza um meio contínuo para ser dividido em diversos elementos que mantém as características do seu original e que são descritos por equações e modelos matemáticos para chegar ao resultado desejado? ${ }^{2}$.

Seguindo este pensamento, a Odontologia aderiu à técnica e a emprega até hoje em diversas especialidades, encontrando na Ortodontia uma grande aplicabilidade de seus recursos ${ }^{3}$. Porém nas outras especialidades, principalmente em implantodontia e prótese dentária este recurso é de grande utilidade, quando se deseja avaliar as tensões oclusais geradas no rebordo alveolar, prevenindo reabsorções ósseas indesejadas ${ }^{4}$.

Com esta ferramenta computacional, complexos comportamentos biomecânicos de próteses e estruturas circunvizinhas podem ser mensurados 5 e, para a análise, é necessária a obtenção de um modelo experimental do objeto a ser estudado, que poderá ser qualquer estrutura do sistema estomatognático ${ }^{2}$. A partir disto um modelo é obtido em CAD (Computer AidDesigner) e inserido no programa de elementos finitos ANSYSTM 10.0 (ANSYS Inc., Canonsburg, PA, USA) e, dentro do programa, o modelo é malhado e atribuído a ele propriedades mecânicas e condições de interface para que possam ser analisadas tensões, deformações e deslocamentos, tanto no modelo completo como nas estruturas que o compõem ${ }^{6}$.

Assim, compreender as falhas que ocorrem na clínica odontológica é necessário, uma vez que os estudos in vitro muitas vezes não conseguem explicar tal situações e os estudos in vivo apresentam dificuldade para serem executados. $O$ uso de estudos in silico mostram-se como uma opção para explicar condições mecânicas odontológicos difíceis de serem reproduzidas por outros tipos de estudos. Com base no exposto, objetiva-se com esta revisão de literatura esclarecer a aplicação da ferramenta de elementos finitos na Odontologia.

\section{REVISÃO DE LITERATURA}

A revisão de literatura foi pautada em uma literatura específica e relevante, ou seja, artigos científicos, teses e livros sobre a temática abordada. Esta literatura foi buscada nas seguintes plataformas: Pubmed.gov, Google.acadêmico, Scielo; Bibliotecas virtuais; iá os descritores utilizados nesta busca foram: Odontologia, Elemento Finito e Bioengenharia.

A aplicabilidade e tomada de resultados através do MEF na Odontologia e a busca por procedimentos operatórios satisfatórios combinaram perfeitamente para uma prática clínica mais moderna, segura e eficiente. Com isso, a implantodontia encontrou a chave para procedimentos operatórios que demandariam manejos extremamente cuidadosos e que com $\circ$ método deixaram $\circ$ pós-operatório seguro e com menos chances de complicações ${ }^{6,7}$.

Portanto, o método dos elementos finitos pode ser realizado antes de um estudo de laboratório, como forma de projetar e conduzir uma pesquisa, para previnir possíveis erros, sendo sua complexidade variável de acordo com as estruturas modeladas. A técnica frequentemente utilizada em estudos de bioengenharia é chamado Bio-CAD, e consiste na obtenção de um modelo virtual geométrico de uma estrutura a partir de referências anatômicas ${ }^{8}$.

Vem se tornado rotina na Odontologia o uso deste método associado a implantodontia, com altos índices de sucesso ${ }^{9}$. Os conceitos de osseointegração são os mais importantes quando se fala em implante dentário e que é definido como o contato direto do tecido ósseo com a superfície de um implante em plena função ao nível de microscopia óptica, sem interposição de tecido fibroso. Neste contexto 
estrutural e funcional $\circ$ método matemático vem auxiliar na simulação de condições em que $\circ$ implante mucossuportado seria inviável e colocá-lo em uma condição de viabilidade ${ }^{10}$.

Com relação às próteses implantossuportadas, é importante avaliar os elementos que são colocados sobre os implantes. O tipo de material para o recobrimento, assim como infraestrutura, anatomia e dimensões em relação ao implante são preponderantes para o sucesso ou fracasso reabilitador ${ }^{7}$. Levando em conta as cargas tensionais sofridas pela prótese sobre implante, é possível identificar como as forças são transmitidas e suas intensidades e distribuições ao osso ${ }^{11} \mathrm{com}$ isso a resina acrílica foi mostrada em estudos que apresenta uma absorção maior de tensões como material de revestimento ${ }^{12}$.

Propriedades do material podem ser determinadas por meio de ensaios mecânicos usando uma configuração de dureza Knoop e o módulo de elasticidadepodeser estimado produzindo um método simples e de baixo custo $^{13}$. Isso influencia diretamente nas aplicações de cargas e como as estruturas a suportam. Existem variados tipos de forças externas como tração, compressão, cisalhamento e torque. Para simular estas variações,cargas pontuais são distribuídas através de uma área específica por meio de uma cúspide oposta simulando um dente antagonista e um ponto de aplicação de carga pode resultar em concentrações de alta tensãoem nós (pontos pelos quais se comunicam os elementos) e assim é avaliado o estresse de concentração dos materiais ${ }^{8}$.

A visualização dosresultadospode ser feita por distribuições de dados mostrando o uso de uma escala de cores, em que cada cor corresponde a um intervalo de valores e que com base neste resultado imediato, o operador pode verificar o deslocamento da estrutura, o tipo de movimento que foi realizado, qual região tem um maior deslocamento e até a forma de redistribuiras tensões na estrutura analisada. Portanto, resultados nas análises dos elementos finitos é quase ilimitada e várias vezes torna-se intimidante, porém a facilidade de visualizar e identificar os resultados torna o método bastante acessível para a prática odontológica ${ }^{8}$.

método dos elementos finitos na Odontologia apresenta-se como uma ferramenta de grande valor para o cirurgião-dentista moderno, pois a qualidade dos seus resultados e suas implicações clínicas são extremamente satisfatórias com essa técnica. A mensuraçãode cargas oclusais impostas em diversos tipos de próteses, implantesou resistências de materiais que compõem os mesmos fazem com que $\circ$ profissional tenha um prognóstico favorável para a reabilitação dos seus pacientes.

A partir de um modelo matemático pode-se observar uma subdivisão de elementos constituintes em vários outros que serão descritos por equações e modelos matemáticos. E o que significa isso? A partir do momento em que o cirurgião dentista trabalha com uma equipe multidisciplinar para o uso do MEF, ele dispões de técnicas que não fazem parte do seu cotidiano (equações diferenciais e modelos matemáticos), porém que irão trazer mensurações biomecânicas que não seriam possíveis com métodos convencionais e não trariam os mesmos resultados.

Quando um elemento de estudoé selecionado as tensões desejadas são aplicadas sobre ele, o seu comportamento é descrito por funções algébricas, em que os achados representarão a distribuição das tensões e deformações do modelo experimental ${ }^{2}$. Com esta afirmação é visto que a obtenção de um modelo experimental de um elemento é de fundamental importância para o resultado final, visto que etapas são extremamente importantes e metódicas que vai desde o desenho gráfico, passa pela discretização em elementos finitos, formação da malha e distribuição das informações sobre os nós das malhas e que com isso obteremos os resultados desejados de avaliação ${ }^{8}$.

Por meio do MEF qualquer material ou estrutura dento-maxilo-facial pode ser modelada e os esforços analisados. Nas especialidades odontológicas, encontramos uma de suas principais vantagens que consiste no fato de poder controlar qualquer variável relacionada a um experimento, facilitando a análise dos resultados, proporcionando grandes benefícios às pesquisas científicas, o que remete ao imenso avanço tecnológico que vem para somar na prática odontológica.

Com todos esses resultados, é necessário ter em mente que o método não é totalmente incontestável e que a precisão de seus resultados também possui 
limites de tolerância ${ }^{2}$, porém não põe em questão a sua confiabilidade. As limitações presentes no uso da FEA consistem principalmente na incapacidade de reproduzir todos os detalhes anatômicos, em virtude das especificidades e complexidade das estruturas orais e craniofaciais. A dificuldade e imprecisão na obtenção de informações referentes às propriedades dos materiais também constituem uma deficiência. A análise de elementos finitos é uma ferramenta de extrema valia para o entendimento biomecânico, porém não substitui a realização de estudos laboratoriais e clínicos para fundamentar uma teoria científica. $\bigcirc$ uso do elemento finito contribui na explicação dos achados em estudos científicos. Outro fator importante é que os estudos em 2D são limitados quando comparados aos em $3 D$, por apresentarem uma limitação estrutural ${ }^{8}$.

\section{CONSIDERAÇÕES FINAIS}

Diante do exposto, conclui-se que a Odontologia moderna encontra no método dos elementos finitos uma forma de aprimorar a prática clínica, através de uma ferramenta computacional, para obter resultados que se apresentam como satisfatórios e eficientes nos diversos procedimentos de reabilitação oral e de análises tensionais e estruturais nos materiais dentários, trazendo assim prognósticos favoráveis aos pacientes e satisfação aos mesmos.

\section{CONFLITOS DE INTERESSES}

Nenhum conflito financeiro, legal ou político envolvendo terceiros (governo, empresas e fundações privadas, etc.) foi declarado para nenhum aspecto do trabalho submetido (incluindo mas não limitandose a subvenções e financiamentos, conselho consultivo, desenho de estudo, preparação de manuscrito, análise estatística, etc).

\section{REFERÊNCIAS}

1. Cook RD, Malkus DS, Plesha ME, Witt RJ. Concepts and Applications of Finite Elements Analysis. John Wiley \& Sons, Inc; 2002.

2. Lotti RS, Machado AS, Mazzieiro ET, Landre Júnior J.
Aplicabilidade científica do método dos elementos finitos. Dental Press Ortodon Ortop Facial. 2006; 11 (2):35-43. doi: $10.1590 /$ S1415-54192006000200006

3. Lotti RS, Mazziero ET, Landre Junior J. A influência do posicionamento da alça segmentada em "T" durante o movimento de retração inicial. Dental Press Ortodon. Ortop. Facial. 2006;1 1(3):41-54. doi: 10.1590/S1415$\underline{54192006000300006}$

4. Abrão GM. Análise pelo método de elementos finitos da distribuição de tensões em dentes pilares de próteses parciais removíveis classe I de kennedy associada a implante osseointegrado [Dissertação]. Uberlândia: Faculdade de Odontologia da Universidade Federal de Uberlândia; 2014.

5. Laganá DC. Comportamento biomecânico das estruturas de suporte e da prótese parcial removível de extremidade livre, com encaixe extracoronário rígido e semi-rígido [Tese de livre docência]. São Paulo: Universidade de São Paulo; 1996.

6. Pessoa RS, Oliveira SAG, Golveia VG, Marcantonio Jr E, Vaz LG. Aplicabilidade do método de elementos finitos na implantodontia. Innovations Implant Journal. 2006; 1(1): $41-7$.

7. Vasconcellos $A B$. Estudo das tensöes em prótese parcial fixa livre de metalalocerâmica - método dos elementos finitos [Tese]. São Paulo: Faculdade de Odontologia da Universidade de São Paulo; 2011.

8. Soares CJ, Versluis A, Valdivia ADCM, Bicalho AA, Veríssimo $C$, Barreto $B C F$ et al. Finite element analysis in dentistryimproving the quality of oral health care. In: Moratal D. Finite Element Analysis - From Biomedical Applications to Industrial Developments. Intech. 201 2.2:25-56. doi: 10.5772/37353

9. Prados-Privado M, Prados-Frutos JC, Gehrke SA, Siles MS, José Luis Calvo Guirado JLG, Bea JA. Long-Term Fatigue and Its Probability of Failure Applied to Dental Implants. BioMed Research International. 2016:ID 8927156:1-8. doi: $10.1155 / 2016 / 8927156$

10. Albrektsson T, Brånemark PI, Hansson HA, Lindström J. Osseointegrated titanium implants . Requirements for ensuring a long-lasting, direct bone-to-implant anchorage in man. Acta orthop. Scand. $1981 ; 52(2): 155-70$.

11. Çiftçi Y, Canaya S. The effect of veneering materials on stress distribution in implant-supported fixed prosthetic restorations. The international journal of oral and maxillofacial implants. 2000;15(4):571-82.

12. Shalak R. Biomechanical considerations in osseointegrated prostheses. J prosthet dent. 1983;49(6):843-8.

13. Marshall DB, Noma T, Evans AG. A simple method for determining elastic-modulus-tohardness ratios using Knoop indentation measurements. J Am Ceram Soc. 2006;65(10):c175-c176. doi: 10.1111/i.1151-2916.1982. tb10357.x 